\title{
De Novo Design of Type II Topoisomerase Inhibitors as Potential Antimicrobial Agents Targeting a Novel Binding Region
}

Kyle M. Orritta ${ }^{\mathrm{a}}$, Juliette F. Newell ${ }^{\mathrm{a}}$, Thomas Germe ${ }^{\mathrm{b}}$, Lauren R. Abbott ${ }^{\mathrm{b}, 1}$, Holly L. Jackson ${ }^{a}$, Benjamin K. L. Burya, Anthony Maxwellb*, Martin J. McPhilliea ${ }^{a \star}$, Colin W.

G. Fishwick ${ }^{\mathrm{a}}$

a School of Chemistry, University of Leeds, Leeds, LS2 9JT, United Kingdom

b Dept. Biological Chemistry, John Innes Centre, Norwich Research Park, Norwich, NR4 7UH, United Kingdom

${ }^{1}$ Dept. of Molecular and Cell Biology, University of Leicester, Leicester, LE1 7RH, United Kingdom

*Corresponding authors: Tony.Maxwell@jic.ac.uk, M.J.McPhillie@leeds.ac.uk, C.W.G.Fishwick@leeds.ac.uk

\section{Abstract}

By 2050 it is predicted that antimicrobial resistance will be responsible for 10 million global deaths annually, costing the world economy $\$ 100$ trillion. Clearly, strategies to address this problem are required as bacterial evolution is rendering our current antibiotics ineffective. The discovery of an allosteric binding site on the established antibacterial target DNA gyrase offers a new medicinal chemistry strategy, as this site is distinct from the fluoroquinolone-DNA site binding site. Using in silico molecular design methods, we have designed and synthesised a novel series of biphenyl-based inhibitors inspired by the published thiophene allosteric inhibitor. This series was evaluated in vitro against $E$. coli DNA gyrase, exhibiting $I_{50}$ values in the low micromolar range. The structure-activity relationship reported herein suggests insights to further exploit this allosteric site, offering a pathway to overcome fluoroquinolone resistance.

Keywords DNA gyrase, antimicrobial resistance, structure-based molecular design, de novo design, allosteric inhibitors 
The evolution of antibiotic resistance poses an enormous threat to human health. The discovery of penicillin in 1928 heralded the beginning of the antibiotic era, revolutionising the treatment of bacterial infections. Antibiotics then became a staple in modern medical procedures such as surgery and organ transplantation. However, following the golden period of antimicrobial drug discovery between the 1960s and 1980s, a decline in novel antibiotic FDA approval coupled with a rise in antibiotic resistance (AMR), has led to a growing increase in the number of untreatable bacterial infections. ${ }^{1}$

DNA gyrase and topoisomerase IV are essential bacterial type II topoisomerases that control DNA topology during DNA replication, transcription and other DNA-associated processes. ${ }^{2,} 3$ DNA gyrase introduces negative supercoils into bacterial DNA via an ATP-dependent mechanism, whereas topoisomerase IV primarily eliminates DNA entanglements that occur during DNA replication. Both enzymes are composed of two proteins, coded for by the gyrA and gyrB genes for DNA gyrase, and the parC and parE genes in the case of topoisomerase IV. 4, 5 These two protein assemblies are composed of four protein subunits, forming heterotetrameric complexes: $A_{2} B_{2}$ for DNA gyrase, and $\mathrm{C}_{2} \mathrm{E}_{2}$ for topoisomerase IV. They are well-documented targets for antimicrobial therapy, with the fluoroquinolones being renowned for possessing a "dual-targeting" mechanism with the possibility of inhibiting both enzymes simultaneously. Dual-targeting is an attractive prospect for antimicrobial drug discovery, as inhibition of two enzymes simultaneously presents bacteria with a significant challenge towards resistance evolution. ${ }^{6,7}$

DNA gyrase and topoisomerase IV possess a high degree of sequence and structural similarity, which favours the dual-targeting approach. They possess limited sequence similarity to that of human topoisomerase II which allows for the design of selective inhibitors for these bacterial topoisomerase enzymes over the human topoisomerase II. 8

The more established sites on the topoisomerases for the interaction of inhibitors are associated with their DNA- and ATP-binding sites. ${ }^{5} 9$ In the case of the fluoroquinolones, these drugs bind to the enzyme-DNA complex and effectively "trap" the bound DNA within the enzyme by forming key interactions within the DNA-binding site via a water-metal ion bridge. ${ }^{10}$ However, despite the success of "dual-targeting" agents such as the fluoroquinolones (FQs), as well as the relatively slow rate at which 
bacterial resistance to these drugs has occurred, resistance within the clinic is growing. ${ }^{11}$ There are concerns that eventually, these antibacterial drugs may become ineffective.

Resistance to fluoroquinolones has developed largely due to point mutations developing in nature and have led to an increasing resistance to fluoroquinolones in recent decades. Point mutations within the gyrA (e.g. S81, S83, D87 and E85; Escherichia coli numbering) and gyrB (e.g. N426 and K447) genes of DNA gyrase, as well as the parC (e.g. S79, D83 and E84) and parE (e.g. D435) genes of topoisomerase IV contribute to said antimicrobial resistance. ${ }^{11}$ The discovery of potent and novel antimicrobial agents that either bind to alternative regions within the topoisomerases or operate via different mechanisms is therefore paramount in combatting the rise of bacterial resistance.

Allosteric binding sites offer promising, alternative mechanistic types of enzyme inhibition. Chan et al. reported one such example within a Staphylococcus aureus DNA gyrase structure containing a thiophene-carboxamide inhibitor (1). ${ }^{12,13}$ Inspection of the co-crystal structure revealed the inhibitor to be bound within a pocket between the GyrA and GyrB subunits. This region is remote from the fluoroquinolone binding site. Inhibitor 1 ( $\mathrm{IC}_{50}: 0.30 \mu \mathrm{M}$, E. coli gyrase) adopts a conformation within this pocket involving the formation of key interactions with residues R630 and E634 (S. aureus numbering), with the amide carbonyl of the inhibitor acting as a hydrogen bond acceptor for the neighbouring arginine residue, and the terminal amine of the ligand acting as a hydrogen bond donor for the neighbouring glutamate residue (Figure 1). The compound inhibits the supercoiling ability of gyrase, as well as stabilising gyrasedependent DNA cleavage. ${ }^{12}$ Unfortunately, the development of inhibitor 1 was terminated due to observed in vivo toxicity issues, although a later publication by the same group described further examples of fused heterocycles replacing or incorporating the thiophene. ${ }^{13}$ 

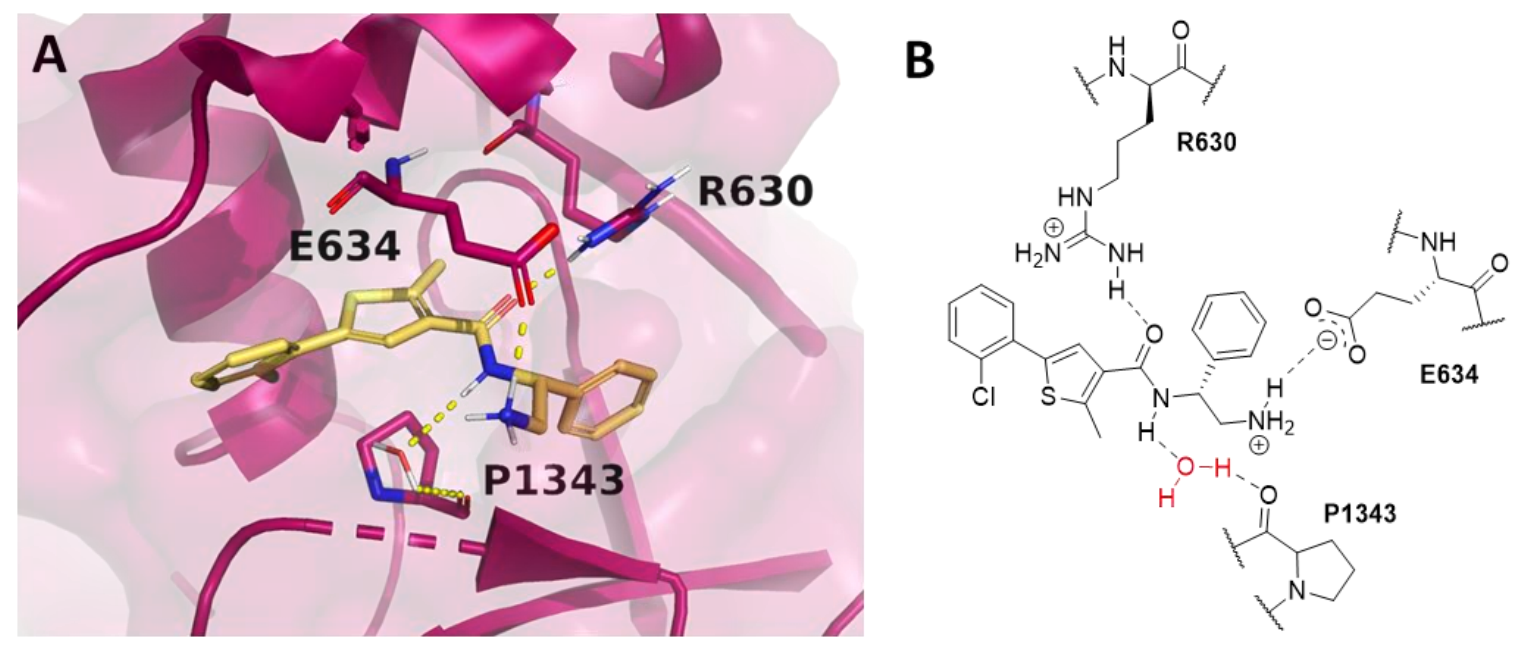

Figure 1. (A) Inhibitor 1 (gold) within the allosteric site of $S$. aureus DNA gyrase (5NPP) ${ }^{12}$. Key hydrogen bonding interactions are shown as yellow dashes to DNA gyrase residues (purple and labelled). Note, the water-mediated hydrogen bond between the amide $\mathrm{NH}$ and P1343. (B) 2D schematic of panel $\mathrm{A}$, highlighting the conserved water in red.

The principals of structure-based ligand design were introduced in the 1980s. ${ }^{14}$ Following significant developments in technological processing power, in silico software, and the availability of high-resolution crystal structures, alternative computational methods to high-throughput screening (HTS) became commonplace to investigate protein target sites and initiate drug discovery projects. SPROUT ${ }^{15}$ is a program used for de novo structure-based molecular design, using a fragment-based approach to design novel scaffolds that can then be ranked by predicted binding affinity. It has been used to design inhibitors of a number of enzymes derived from infectious diseases. ${ }^{16-20}$ Using the co-crystal structure of $S$. aureus DNA gyrase with inhibitor 1 (PDB ID: 5NPP) ${ }^{12}$, we aimed to design novel compounds which bind within the allosteric site and may offer hope in combating the threat of fluoroquinolone resistance by retaining gyrase activity against FQ-resistant strains.

Based on the X-ray conformation of compound 1 from 5NPP, we decided to utilise the three key hydrogen bonding interactions in our molecular designs (R630, E634 and the water-mediated to P1343). This allowed the 'right-hand' portion of $\mathbf{1}$ (as drawn in Figure 1) to be kept consistent within the SPROUT software, and the hydrophobic lefthand portion varied by the steric constraints of the binding cavity. Amongst the initial results, a biphenyl molecular scaffold, as represented by compound 2 , was a synthetically attractive candidate to test the potential of our de novo design approach to targeting this binding site. In summary, compound 2 was designed to retain the 
same hydrogen bonding interactions as observed for compound 1, but the biaryl portion was predicted to make an additional favourable interaction with R1342 via a cation-m interaction (Figure 2).
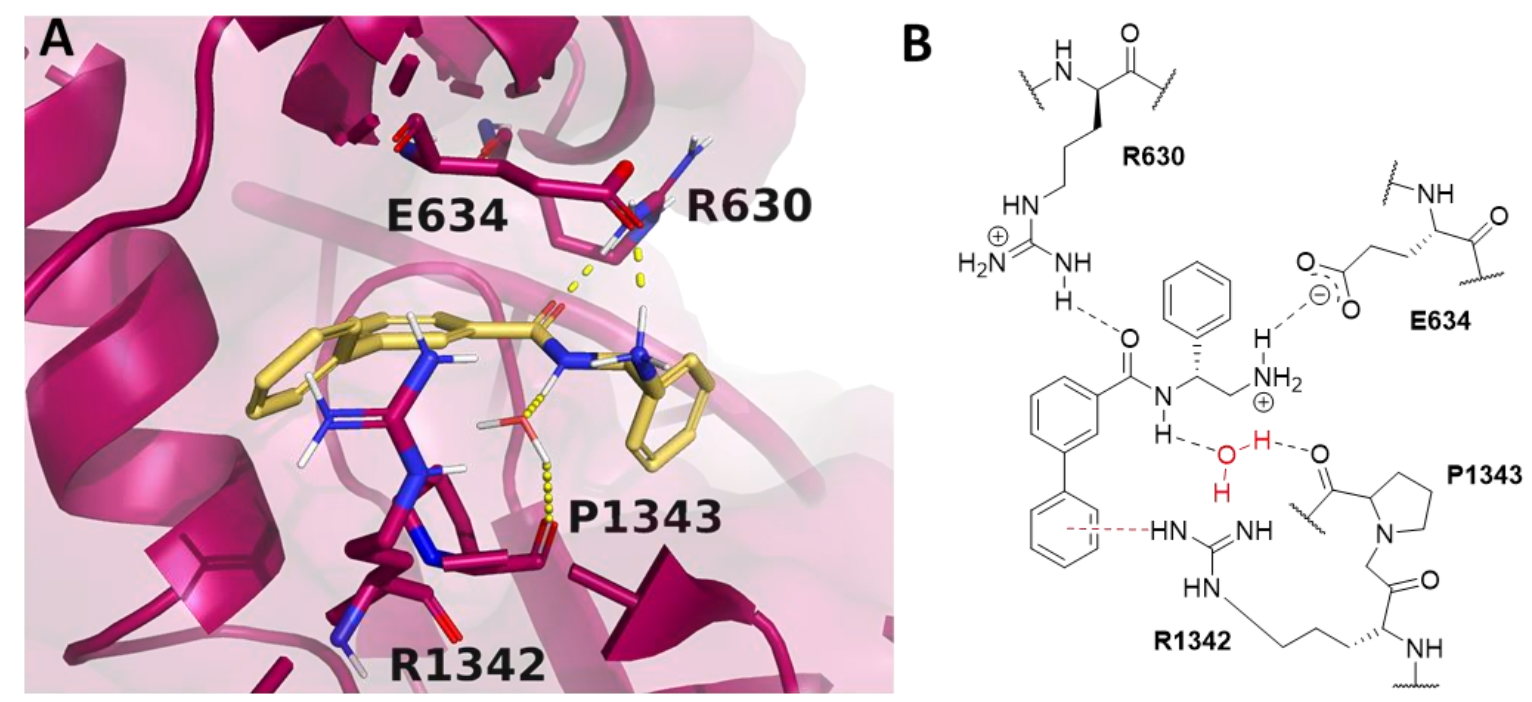

Figure 2. (A) Novel biphenyl inhibitor design 2 (gold) modelled within the allosteric site of $S$. aureus DNA gyrase (5NPP) ${ }^{12}$. Key hydrogen bonding interactions shown in yellow and key residues (purple) are labelled. (B) 2D schematic of panel $A$.

Compound 2 was synthesised and tested in an in vitro E. coli DNA gyrase supercoiling assay, revealing 2 to be a moderate inhibitor of $E$. coli gyrase with an $\mathrm{IC}_{50}$ value of 60 $\mu \mathrm{M}$ (chemical synthesis and biochemical assays described below). With this encouraging result in mind, we endeavoured to explore the structure-activity relationships (SARs) by varying the substitution pattern on the biaryl moiety.

To help guide the design of these substituted analogues, the docking module Glide within the Schrödinger Maestro software package ${ }^{21}$ was used. Various functional groups were computationally added to the biphenyl ring system, and these compounds were then docked within the allosteric site of the 5NPP co-crystal structure in order to explore the steric and electronic characteristics within the hydrophobic cavity. Compounds containing electron-donating groups on the biaryl, such as $\mathbf{3}$ and $\mathbf{4}$ (Table 1), were predicted to display enhanced binding to the enzyme, with higher docking scores (a measure of predicted binding affinity). This was rationalised as increasing the magnitude of the cation-m interaction due to enhanced electronic attraction. Certain substituents were predicted to induce a twist in the structure, compared to the docked pose of compound 2, through increasing the torsion angle between the two phenyl rings. This ring twist was also observed in the 5NPP structure containing 
thiophene 1, and therefore likely required or tolerated for bioactivity. The ring twist was calculated to be $43^{\circ}$ in the case of compound 1 , and $61^{\circ}$ for compound 2 .

In addition, a number of other substituted molecules, containing both electronwithdrawing (molecules 11 and 12), halogen (molecules 9 and 10), alkyl (molecule 5), and combinations of some of these substituents (molecules 6 and 7) were also prepared to explore the SARs within this inhibitor series.

The synthesis of these compounds was readily achieved (Scheme 1). The commercially available (S)-2-amino-1-phenylethanol starting material (14) underwent Boc-protection ahead of a Mitsunobu reaction in the form of a Gabriel synthesis to switch the secondary alcohol for a phthalimide functionality, leading to inversion of stereochemistry. This was followed by cleavage of the phthalimide with hydrazine to result in primary amine 17. Compound 17 was then coupled to 3-bromobenzoyl chloride to form the key amide linker, with the 3-bromo position of 18 primed for the subsequent Suzuki-Miyaura coupling of the various substituted boronic acids. The Boc-protecting group was then removed using strong acid to give compounds 2-13.
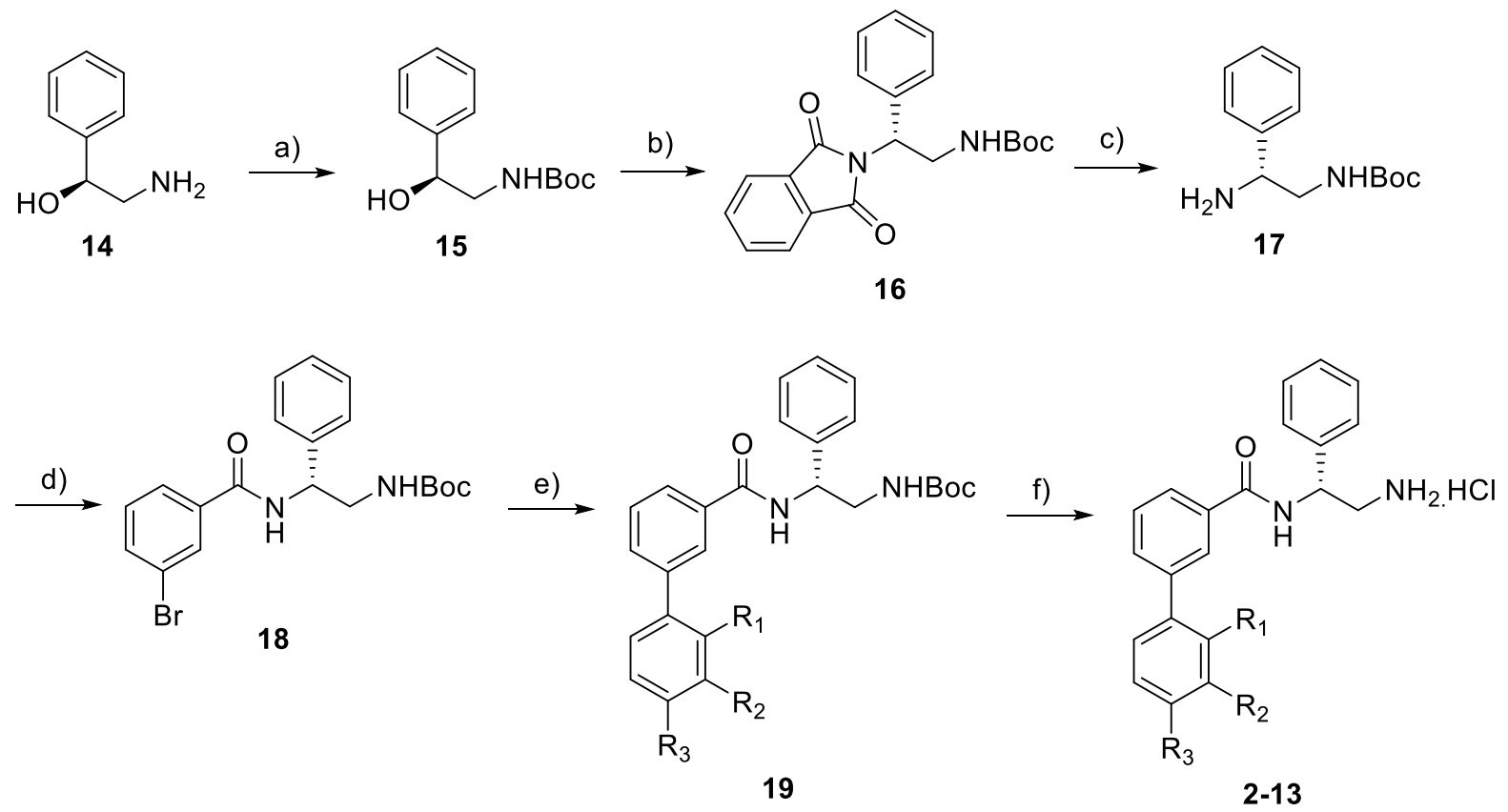

Scheme 1. Reagents and conditions: a) $\mathrm{Boc}_{2} \mathrm{O}$, THF, RT, 1 hour, 89\%. b) Phthalimide, PPh $\mathrm{P}_{3} \mathrm{DEAD}$, THF, $\mathrm{N}_{2}, 0^{\circ} \mathrm{C}-\mathrm{RT}, 18$ hours, $63 \%$. c) $\mathrm{N}_{2} \mathrm{H}_{4} . \mathrm{H}_{2} \mathrm{O}$, EtOH, $60^{\circ} \mathrm{C}, 4$ hours, $74 \%$. d) 3-bromobenzoyl chloride, NEt 3 , DCM, N2, RT, 22 hours, 77\%. e) substituted boronic acid, Pd(PPh $)_{4}$, Propanol, 2M $\mathrm{Na}_{2} \mathrm{CO}_{3}$ aqueous solution, $\mathrm{N}_{2}$, reflux, $20-35 \%$. f) $4 \mathrm{M} \mathrm{HCl}$ in dioxane, $\mathrm{RT}, 65-93 \%$.

The compounds were tested in a DNA supercoiling assay with E. coli DNA gyrase using gel electrophoresis. This is a semi-quantitative assay which is used to identify 
potent compounds relative to the positive control (usually ciprofloxacin) and establish the SARs within a compound series. Compounds were tested in duplicate.

Pleasingly, as predicted, compounds containing electron-donating substituents (3, 4, 6, 7, and 8) showed enhanced inhibitory potency versus the unsubstituted system $\mathbf{2}$, with 6 displaying a 3-fold increase in binding affinity $\left(\mathrm{IC}_{50} ; 17 \mu \mathrm{M}\right)$. Compound 6 was also tested in vitro against $E$. coli topoisomerase IV, to establish its dual targeting potential but unfortunately it was observed to be inactive (Table 2S supplementary information). Interestingly, in the case of compound 7, the introduction of a fluorine atom at the 4-position of the terminal ring resulted in a $\sim 3$-fold reduction in biological activity compared to that observed for isomer $\mathbf{6}$. This is rationalised by the clashing of the fluorine atom in $\mathbf{7}$ with the wall of the binding cavity. Substituents at this position were therefore avoided.

Analysis of the biological data for compounds 9-12 revealed that substitution on the terminal ring of the biaryl moiety is generally tolerated, with moderate increases in affinity compared to unsubstituted system 2 . These include halogenated (9 and 10) and 2-acetyl (12) compounds. The incorporation of stronger electron-withdrawing substituents such as 2-trifluoromethyl (5) or 3-nitro (11) led to a decrease in observed affinity compared to the unsubstituted system 2 , supporting the docking hypothesis. Notably, when $\mathrm{R}$ is a bromine atom (13), activity dropped 5-fold compared to when $\mathrm{R}$ is an unsubstituted phenyl ring (2). Compound 13 is predicted to retain the hydrogen bonding network as for the biaryl analogues, alluding to the importance of the hydrophobic contributions to binding affinity for this compound series. 

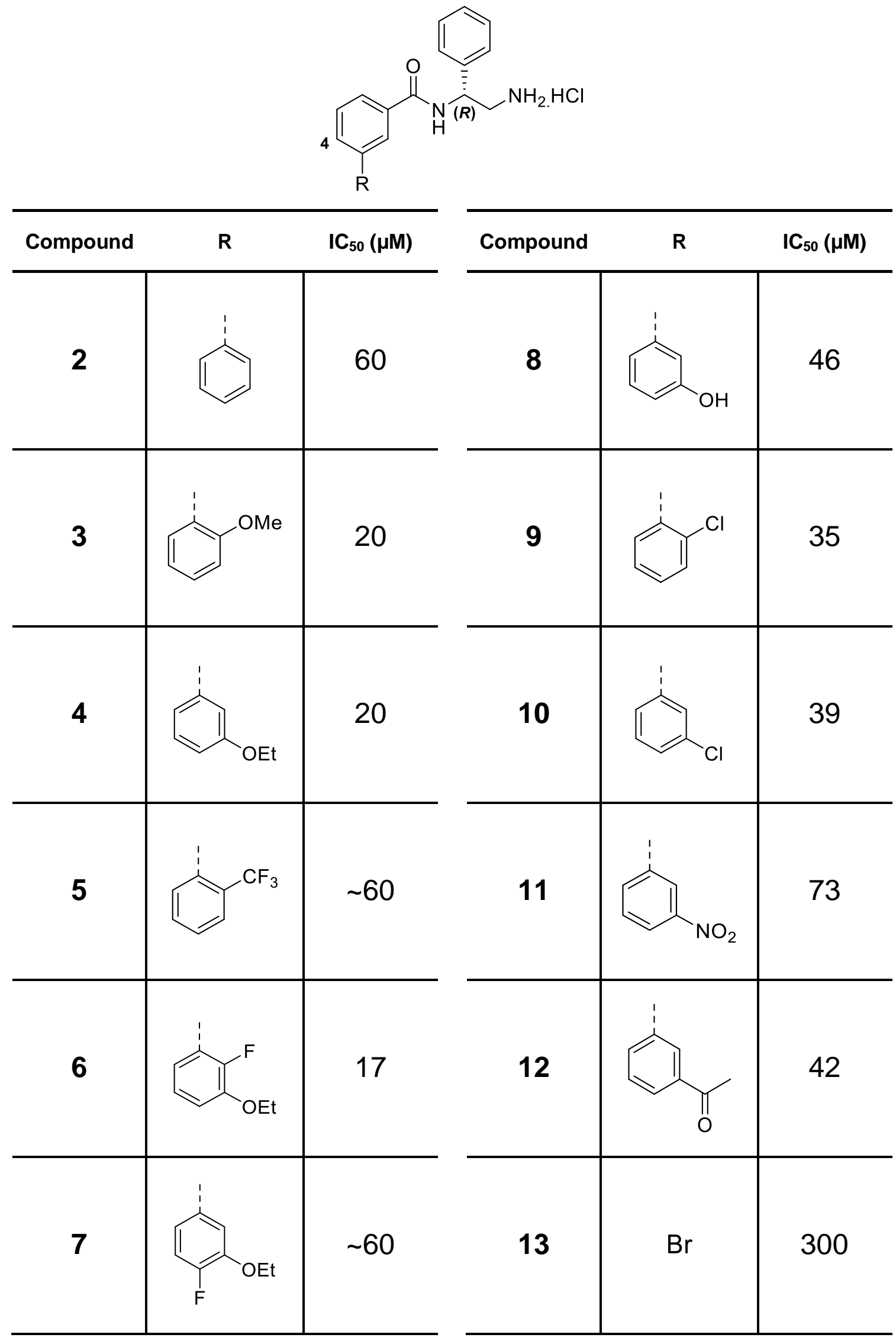

Table 1. Investigation into the SAR of the substituted biphenyl compounds and their activity against $E$. coli DNA gyrase in a supercoiling assay. 
With a broad SAR profile in hand, further analogues were synthesised which would probe the hydrophobic and steric requirements of the hydrophobic cavity, investigate the chirality at the asymmetric centre and explore the addition of substituents to the chiral phenyl group.

The methyl and chloro substituents on compound $\mathbf{1}$ appeared to optimally fill small pockets within hydrophobic cavity. We attempted to mimic this by introducing methyl groups at the 4-position on the benzamide ring and at the 5-position on the terminal phenyl respectively, using the synthetic steps outlined in scheme 1 (compounds 2022, Table 1S supplementary information). These changes proved detrimental to activity $\left(\mathrm{IC}_{50} ; \sim 60 \mu \mathrm{M}\right)$ likely due to either an unfavourable binding conformation or to unfavourable steric clashes with gyrase. Therefore, vector growth into the hydrophobic pocket will in future more closely mimic that of compound $\mathbf{1}$.

The $R$-chirality present in thiophene $\mathbf{1}$ was required for binding to the allosteric site by positioning the chiral phenyl moiety in a favourable vector. ${ }^{12}$ The $S$-isomer of 1 was reported as being $\sim 30$-fold less active..$^{12}$ In order to ascertain whether the $R$-chirality was crucial for our compound series, a number of $S$-chirality compounds (23-25) were prepared using an analogous sequence to that shown in Scheme 1, but starting with (R)-2-amino-1-phenylethanol (Table 3). As expected, these 'chirally-reversed' compounds showed a general decrease in activity compared to their enantiomeric $R$ counterparts (23 vs. 2 ; 24 vs. 3 ; and 25 vs. 4), although the fold-decrease was only significant for compound 25 ( 9-fold). 
<smiles>[R]c1cccc(C(=O)NC([R])CN)c1</smiles>

\begin{tabular}{lll|l}
\hline Compound & \\
\hline 23 &
\end{tabular}

Table 2. Further SAR exploring the role of chirality and substitution on the phenyl ring. $\mathrm{IC}_{50}$ values were determined against $E$. coli DNA gyrase in a supercoiling assay. 
Finally, in order to explore the role of substitution on the $R$-phenyl moiety within the compound series, a number of analogues were synthesised where the phenyl ring was replaced by a phenol. This ring system was selected due to favourable molecular modelling predictions which suggested that this solvent-exposed region of the pocket could potentially offer another bonding interaction. We aspired to form an additional stabilising hydrogen bond to R630 via an intermolecular bridge with a labile water molecule. For these analogues, an optically pure starting material was not commercially available, and so racemic starting materials were used during their synthesis (see Scheme 1S in supplementary material for adapted synthetic route). Five analogues (26-30) were synthesised and tested in vitro, with all being moderate inhibitors of DNA gyrase, demonstrating the scope for further derivatisation in this region.

In summary, the use of a de novo molecular design approach has successfully led to the identification of a new series of inhibitors targeting a novel allosteric binding site within bacterial DNA gyrase. Several compounds were synthesised based on molecular modelling hypotheses, and these were tested in vitro against DNA gyrase using a supercoiling assay. This led to compound $\mathbf{6}$ which had an improved $\mathrm{IC}_{50}$ value of $17 \mu \mathrm{M}$ compared to our initial parent compound 2. Further work is underway to improve this biaryl series, as well as develop alternative scaffolds for this allosteric site. Our approach demonstrates that a combination of traditional medicinal chemistry and in silico molecular modelling can predict bioactive trends for this target and may lay the foundations to identify future novel gyrase antibacterial agents.

Author contributions $\mathrm{KMO}$ and MJM designed the compounds. KMO, JFN, BKLB and HLJ synthesised the compounds. TG and LRA evaluated the compounds. MJM, AM and CWGF designed the study. KMO, MJM, AM and CWGF wrote the manuscript. All authors contributed to the editing of the manuscript.

Funding sources KMO was awarded a Leeds Doctoral Scholarship in 2018.

Acknowledgments We greatly acknowledge the help and assistance provided by the technical staff within the School of Chemistry at Leeds and the Department of Biological Chemistry at John Innes Centre. We thank Inspiralis for screening compounds in the wider context of the project, and Dr Ben Bax for helpful discussions. 


\section{References}

1. Conly, J. M.; Johnston, B. L., Where are all the new antibiotics? The new antibiotic paradox. The Canadian Journal of Infectious Diseases \& Medical Microbiology 2005, 16 (3), 159-160.

2. Bush, N. G.; Evans-Roberts, K.; Maxwell, A., DNA Topoisomerases. EcoSal Plus 2015, 6 (2).

3. McKie, S. J.; Neuman, K. C.; Maxwell, A., DNA topoisomerases: Advances in understanding of cellular roles and multi-protein complexes via structure-function analysis. Bioessays 2021, 43 (4), e2000286.

4. Hooper, D. C.; Jacoby, G. A., Topoisomerase Inhibitors: Fluoroquinolone Mechanisms of Action and Resistance. Cold Spring Harb Perspect Med 2016, 6 (9).

5. Bush, N. G.; Diez-Santos, I.; Abbott, L. R.; Maxwell, A., Quinolones: Mechanism, Lethality and Their Contributions to Antibiotic Resistance. Molecules 2020, 25 (23).

6. Tse-Dinh, Y. C., Targeting bacterial topoisomerases: how to counter mechanisms of resistance. Future Med Chem 2016, 8 (10), 1085-100.

7. Pan, X. S.; Fisher, L. M., DNA gyrase and topoisomerase IV are dual targets of clinafloxacin action in Streptococcus pneumoniae. Antimicrob Agents Chemother 1998, 42 (11), 2810-6.

8. Pommier, Y.; Leo, E.; Zhang, H.; Marchand, C., DNA topoisomerases and their poisoning by anticancer and antibacterial drugs. Chem Biol 2010, 17 (5), 42133.

9. Maxwell, A.; Bush, N. G.; Germe, T.; McKie, S. J.; In Fong, I. W.; Shlaes, D.; Drlica, K., Antimicrobial resistance and

implications for the 21st century. Springer: Switzerland, 2018.

10. Aldred, K. J.; McPherson, S. A.; Turnbough, C. L., Jr.; Kerns, R. J.; Osheroff, N., Topoisomerase IV-quinolone interactions are mediated through a water-metal ion bridge: mechanistic basis of quinolone resistance. Nucleic acids research 2013, 41 (8), 4628-4639.

11. Redgrave, L. S.; Sutton, S. B.; Webber, M. A.; Piddock, L. J. V., Fluoroquinolone resistance: mechanisms, impact on bacteria, and role in evolutionary success. Trends in Microbiology 2014, 22 (8), 438-445.

12. Chan, P. F.; Germe, T.; Bax, B. D.; Huang, J.; Thalji, R. K.; Bacqué, E.; Checchia, A.; Chen, D.; Cui, H.; Ding, X.; Ingraham, K.; McCloskey, L.; Raha, K.; Srikannathasan, V.; Maxwell, A.; Stavenger, R. A., Thiophene antibacterials that allosterically stabilize DNA-cleavage complexes with DNA gyrase. Proceedings of the National Academy of Sciences 2017, 114 (22), E4492.

13. Thalji, R. K.; Raha, K.; Andreotti, D.; Checchia, A.; Cui, H.; Meneghelli, G.; Profeta, R.; Tonelli, F.; Tommasi, S.; Bakshi, T.; Donovan, B. T.; Howells, A.; Jain, S.; Nixon, C.; Quinque, G.; McCloskey, L.; Bax, B. D.; Neu, M.; Chan, P. F.; Stavenger, R. A., Structure-guided design of antibacterials that allosterically inhibit DNA gyrase. Bioorg Med Chem Lett 2019, 29 (11), 1407-1412. 
14. Mauser, H.; Guba, W., Recent developments in de novo design and scaffold hopping. Curr Opin Drug Discov Devel 2008, 11 (3), 365-374.

15. Gillet, V.; Johnson, A. P.; Mata, P.; Sike, S.; Williams, P., SPROUT: A program for structure generation. Journal of Computer-Aided Molecular Design 1993, 7 (2), 127-153.

16. McPhillie, M. J.; Trowbridge, R.; Mariner, K. R.; O’Neill, A. J.; Johnson, A. P.; Chopra, I.; Fishwick, C. W. G., Structure-Based Ligand Design of Novel Bacterial RNA Polymerase Inhibitors. ACS Medicinal Chemistry Letters 2011, 2 (10), 729-734.

17. Cowen, D.; Bedingfield, P.; McConkey, G. A.; Fishwick, C. W. G.; Johnson, A. P., A study of the effects of substituents on the selectivity of the binding of $\mathrm{N}$ arylaminomethylene malonate inhibitors to DHODH. Bioorganic \& Medicinal Chemistry Letters 2010, 20 (3), 1284-1287.

18. Cain, R.; Brem, J.; Zollman, D.; McDonough, M. A.; Johnson, R. M.; Spencer, J.; Makena, A.; Abboud, M. I.; Cahill, S.; Lee, S. Y.; McHugh, P. J.; Schofield, C. J.; Fishwick, C. W. G., In Silico Fragment-Based Design Identifies Subfamily B1 Metallo- $\beta$-lactamase Inhibitors. J Med Chem 2018, 61 (3), 1255-1260.

19. Narramore, S.; Stevenson, C. E. M.; Maxwell, A.; Lawson, D. M.; Fishwick, C. W. G., New insights into the binding mode of pyridine-3-carboxamide inhibitors of E. coli DNA gyrase. Bioorg Med Chem 2019, 27 (16), 3546-3550.

20. Yule, I. A.; Czaplewski, L. G.; Pommier, S.; Davies, D. T.; Narramore, S. K.; Fishwick, C. W., Pyridine-3-carboxamide-6-yl-ureas as novel inhibitors of bacterial DNA gyrase: structure based design, synthesis, SAR and antimicrobial activity. Eur $J$ Med Chem 2014, 86, 31-8.

21. Schrödinger Maestro, LLC: New York, 2019. 